\title{
Performance comparison of automatic peak detection for signal analyser
}

\author{
Teh Yi Jun ${ }^{1}$, Asral Bahari Jambek ${ }^{2}$, Uda Hashim ${ }^{3}$ \\ ${ }^{1,2}$ School of Microelectronic Engineering, Universiti Malaysia Perlis, Perlis, Malaysia \\ ${ }^{3}$ Insituite of Nano Electronic Engineering, Universiti Malaysia Perlis, Perlis, Malaysia
}

\begin{tabular}{|c|c|}
\hline Article Info & ABSTRACT \\
\hline Article history: & The aim of this paper is to propose a new peak detection method for a \\
\hline Received Oct 18, 2018 & (M-ATPD). M-ATPD evolves out of ATPD with a focus on reducing \\
\hline Revised Nov 23, 2018 & computational time. The proposed method replaces the clustering threshold \\
\hline Accepted Dec 24, 2018 & $\begin{array}{l}\text { calculation in ATPD with a standard deviation threshold calculation. M- } \\
\text { ATPD reduces computational time by } 2 \text { times faster compared to ATPD for }\end{array}$ \\
\hline Keywords: & $\begin{array}{l}\text { control signal and } 8.65 \text { times faster compared to ATPD for raw biosignals. } \\
\text { Modified ATPD also shows a slight improvement in terms of detection error, }\end{array}$ \\
\hline $\begin{array}{l}\text { Automatic peak detection } \\
\text { Peak detection } \\
\text { Signal analyser }\end{array}$ & $\begin{array}{l}\text { with a decrease of about } 6.66 \% \text { to } 13.33 \% \text { in peak detection of noise signals. } \\
\text { Modified ATPD successfully fixes the error of peak detection on pulse } \\
\text { control signals associated with ATPD. For raw biosignals, in total M-ATPD } \\
\text { achieved } 19.41 \% \text { lower detection error compare to ATPD. }\end{array}$ \\
\hline
\end{tabular}

Copyright $@ 2019$ Institute of Advanced Engineering and Science. All rights reserved.

\section{Corresponding Author:}

Teh Yi Jun,

School of Microelectronic Engineering,

University Malaysia Perlis,

Pauh Putra Campus, 02600 Pauh, Perlis, Malaysia.

Email: kelvinteh90@gmail.com

\section{INTRODUCTION}

Amplitude response is most common in electronic application. Peak detection plays the most important role in analysing amplitude response signals. However, noise will cause false peak detection or report a missing peak. This will decrease the accuracy of peak detection. Hence, intelligent peak detection methods are required to increase the accuracy of analysis. From time to time, peak detection methods are design for general or specific signal, such as automatic multiscale peak detection (AMPD) [1], the automatic chromatographic peak detection (ACPD) [2], the adaptive threshold method (ATM) [3], the peak of Shannon energy envelope (PSEE) [4] and ATPD [5, 6].

AMPD and ATPD are designed for general signal peak detection. ACPD is specifically designed for chromatographic signals. The PSEE is specifically designed for electrocardiogram (ECG) signals, while the ATM is specifically designed for photoplethysmorgraphy signals. Although some peal detection algorithms are designed for specific signals, certain criteria in the algorithm might be applicable to each other due to the similarity of signals.

Besides accuracy, computational complexity is the main concern when designing an algorithm [7]. Computational complexity relates to the direct effect of the efficiency of time and power [8,9]. Our aim is to design a peak detection algorithm for a portable nano-biosensor device, which will require lower power consumption such that the size of the battery and the portable device overall can be reduced. According to the literature, ATPD methods show the lowest detection error with moderate computational timing. Hence, ATPD is proposed to improve computational timing. The rest of the paper is organized as follows. Section II reviews the existing ATPD algorithm. Section III elaborates on the evolution of the ATPD algorithm. The results of our experiment are presented in Section IV, while Section V concludes this paper. 


\section{LITERATURE REVIEW}

Figure 1 is a flow chart for the threshold calculation method in ATPD. At the threshold calculation stage, clustering methods are applied. Initially, the algorithm will load the signal data $(\mathrm{x}[\mathrm{n}])$. Then, the first signal data $(\mathrm{x}[1])$ and the second signal data $(\mathrm{x}[2])$ will be set as a cluster value $1(\mathrm{C} 1)$ and cluster value 2 (C2), respectively. The algorithms will continuously load the next data on a loop in order to compare C1 and $\mathrm{C} 2$, such that the data will grouped into the nearest cluster class and a new cluster value will be calculated from the mean of the cluster class. After a new cluster value is calculated, this will be compared to the last cluster value. The loop will repeat until the differences between the new cluster value and the last cluster value are smaller than those for the termination condition $(\varepsilon)$. Then, the smallest cluster value will be selected as the threshold value. The selected threshold value will be used to determine the peak. The peak is defined when the difference between the trough and the peak is greater than threshold value; otherwise, it will not counted as the peak. By using the threshold value, much of the false peak created by noise will be eliminated.

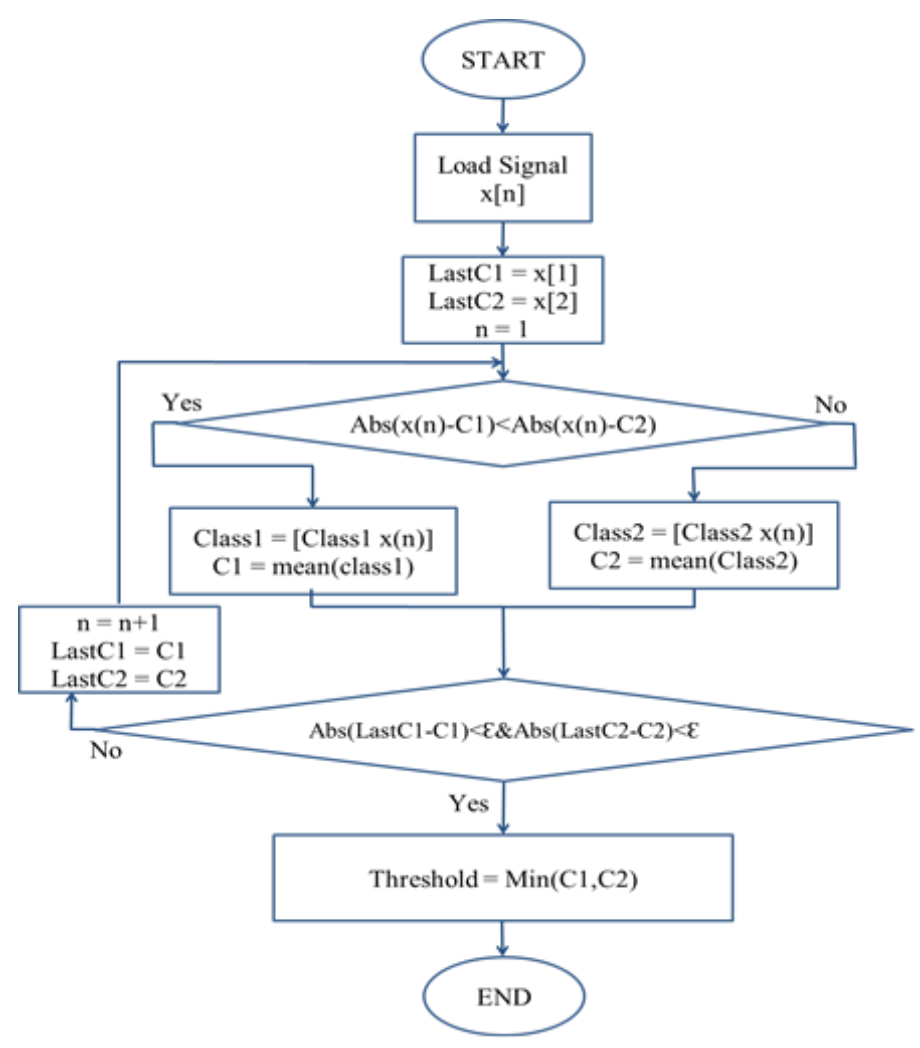

Figure 1. Flow chart for the cluster threshold calculation method

Then, the simulation of ATPD algorithm is conducted using MATLAB. Figure 2 shows the results of peak detection using ATPD. The triangular mark represents the detected peak. The purpose of simulation is to identify the computational time for each stage. Figure 3 shows the profile summary from MATLAB simulation. From the study, the most time-consuming stage is the threshold calculation stage. The time taken to carry out the cluster threshold calculation is $0.334 \mathrm{~s}$ out of a total time of $0.556 \mathrm{~s}$. This shows that more than half of the total time is used for the threshold calculation. Hence, improvements at the threshold calculation stage are required in order to reduce the overall computational time. A new threshold calculation algorithm using standard deviation is proposed. The next section will discuss the new threshold calculation. 


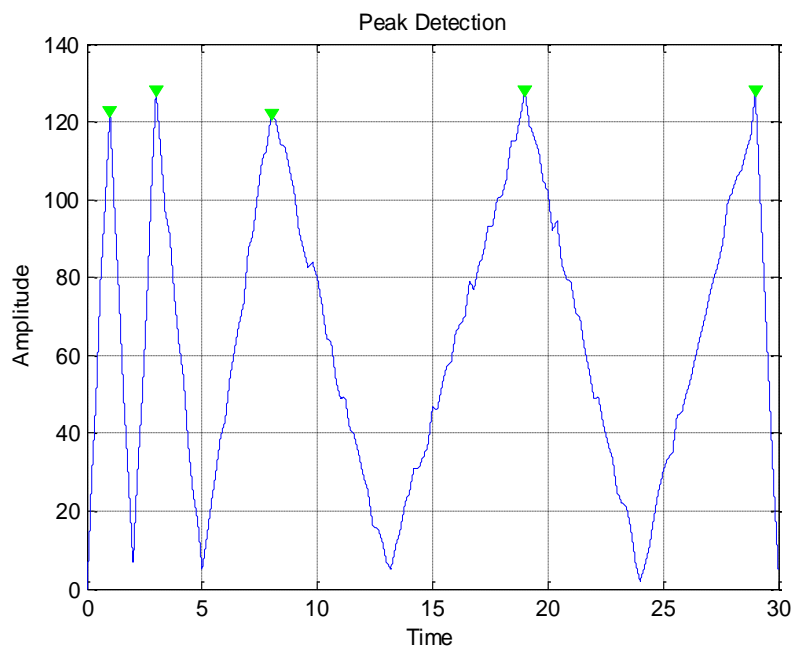

Figure 2. Peak detection using ATPD

\begin{tabular}{|c|c|c|c|c|}
\hline \multicolumn{5}{|c|}{$\begin{array}{l}\text { Profile Summary } \\
\text { Generated 21-Jan-2016 22:01:5 }\end{array}$} \\
\hline Function Name & $\underline{\text { Calls }}$ & Total Time & $\underline{\text { Self Time }}^{*}$ & $\begin{array}{l}\text { Total Time Plot } \\
\text { (dark band = self time) }\end{array}$ \\
\hline ATPD & 1 & $0.555 \mathrm{~s}$ & $0.070 \mathrm{~s}$ & - \\
\hline twoclass & 1 & $0.334 \mathrm{~s}$ & $0.334 \mathrm{~s}$ & 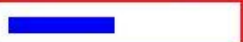 \\
\hline
\end{tabular}

Figure 3. Profile summary of ATPD created by MATLAB simulation

\section{METHODOLOGY}

In Section II, the details of the ATPD algorithm was discussed. In this section, the details of the proposed threshold calculation method will be considered. According to the literature, standard deviation is widely use to estimate the noise level $[10,11]$. Hence, the proposed method is referred to as the standard deviation threshold calculation. The purpose of the proposed new method is to solve the problem of heavy computational complexity at the threshold calculation stage in ATPD. In the proposed method, standard deviation is used to estimate the noise level, which is then adopted as the threshold to eliminate the false peak. The usual purpose of standard deviation is to calculate abnormal data or explain why the data are too different from other data. Figure 4 illustrates the concept of standard deviation. Abnormal data will be outside the range of standard deviation. In real signals, noise peak will occur more frequently than true peaks. Hence, noise peaks will be in the majority, while true peaks be in the minority. As such, the peak within the standard deviation range will be the noise peak, whereas the true peak will be outside the standard deviation range. Figure 5 is a flow chart for the standard deviation threshold calculation method. In the standard deviation threshold calculation, the signal data $(\mathrm{x}[\mathrm{n}])$ will initially be loaded. Then, all the turning points of the loaded signal will be detected, which are shown in Figure 6 as triangular marks. After that, the detected turning points are used to calculate standard deviation for the purpose of estimating the noise level. Lastly, the threshold value will be defined by $\alpha \times \operatorname{sd}$, where $\alpha$ is the coefficient calibrate based on the signal and sd is standard deviation. Firstly, control signal are used to evaluate the M-ATPD. Eighteen control signals are generated consisting of three types of peak: pulse, sinusoidal, and triangular. Each type of peak signal has a best, typical, and worst case. The same control signals are then combined with noise to test the performance of the algorithms. All control signals are generated using Matlab and have a signal length of 30 seconds and a sampling frequency of $640 \mathrm{~Hz}$. 


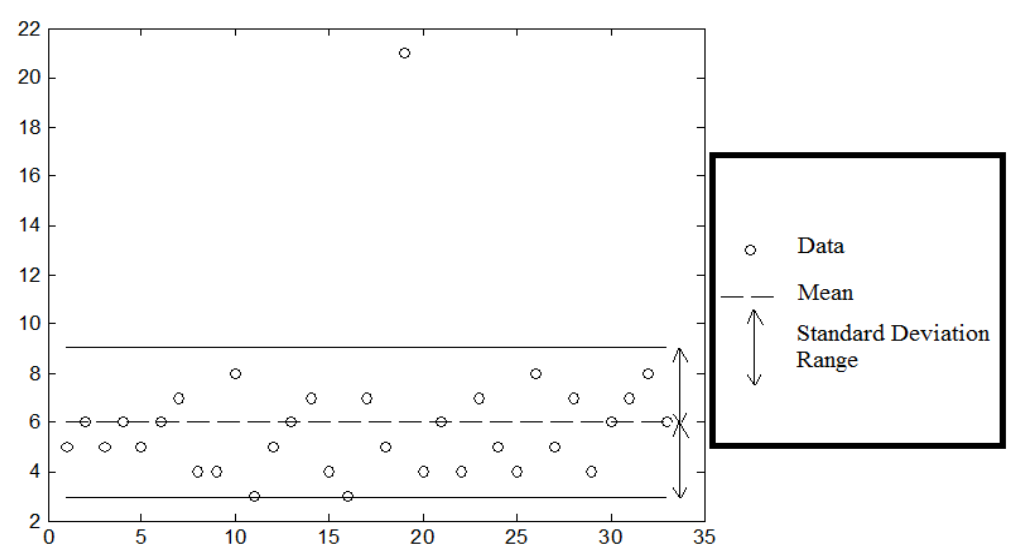

Figure 4. Concept of standard deviation

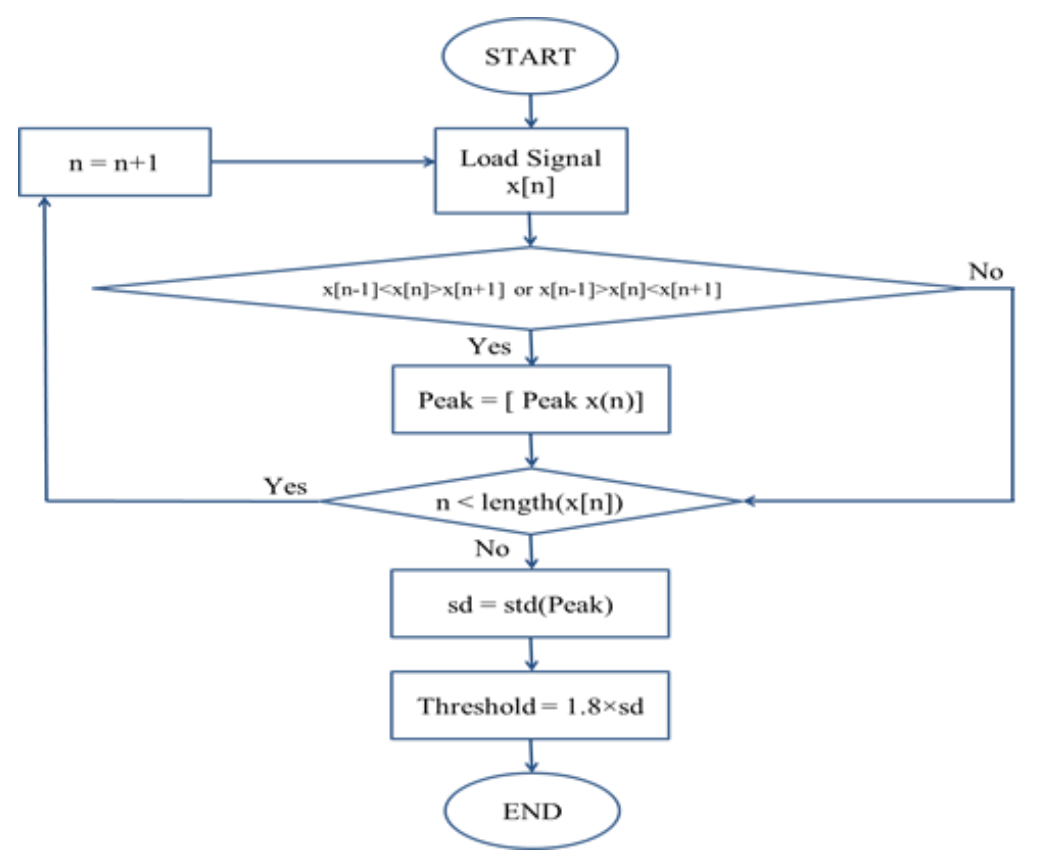

Figure 5. Flow chart for the standard deviation threshold calculation method

Next, several biosignals were chosen: electrocardiogram (ECG), blood pulse (BP), electroencephologram (EEG) and sum of respiration (Resp(sum)) to further evaluation. The raw signals of ECG, BP, EEG and Resp(sum)were applied in the experiment to determine the ability of M-ATPD to analyse various types of signal. The length of each signal is 900000 data in 3600 seconds. M-ATPD required adjustment of the coefficient when applied to different signals. This adjustment of the coefficient was done manually. The coefficients for ECG/BP, EEG, and sum of respiration were set to 4.0, 2.5 and 1.0 respectively. The same signal was then applied using ATPD. The raw signal required a level-shifting process to ensure that ATPD worked correctly with each signal, and this level-shifting process was done manually. Level shifts for ECG, BP, EEG and Resp(sum) were $+250,+300,+100$ and +600 respectively. A comparison was then made to assess the improvements from M-ATPD in comparison with ATPD. The results will be discuss in next section. 


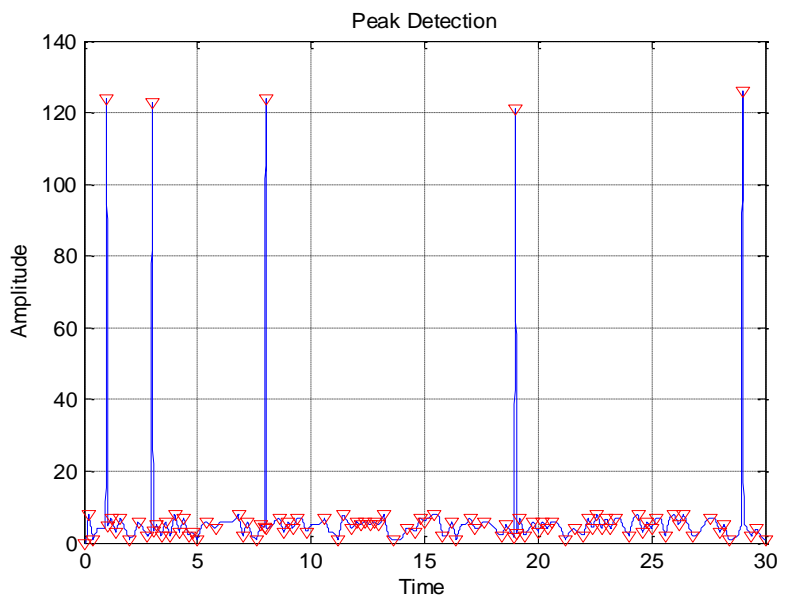

Figure 6. All determined turning point using the standard deviation threshold calculation method

\section{RESULTS AND DISCUSSIONS}

In Section III, the details of the standard deviation threshold calculation method were discussed. This method replaces the original clustering threshold calculation in ATPD. Hence, we have a new peak detection method called M-ATPD. Figure 7 shown peak detection using M-ATPD. Figure 8 shows the profile summary for M-ATPD using MATLAB simulation. Results show that the standard deviation threshold calculation time reduces from $0.334 \mathrm{~s}$ to $0.042 \mathrm{~s}$. In other words, more than $80 \%$ of the time taken for threshold calculation is saved when the standard deviation threshold calculation replaces the cluster threshold calculation. Table 1 summarizes the computational time for M-ATPD and another five studied algorithms using MATLAB simulation. The results show that M-ATPD reduces computational time by $50 \%$ compared to ATPD. M-ATPD also has the lowest computational time, along with the ATM. Computational time is direct proportional to power consumption, which means that M-ATPD may able to reduce power consumption by $50 \%$. In turn, great improvements have been achieved in term of reducing computational complexity. Table 2 summarizes the accuracy of peak detection using M-ATPD when compared with another five studied algorithms using MATLAB simulation.

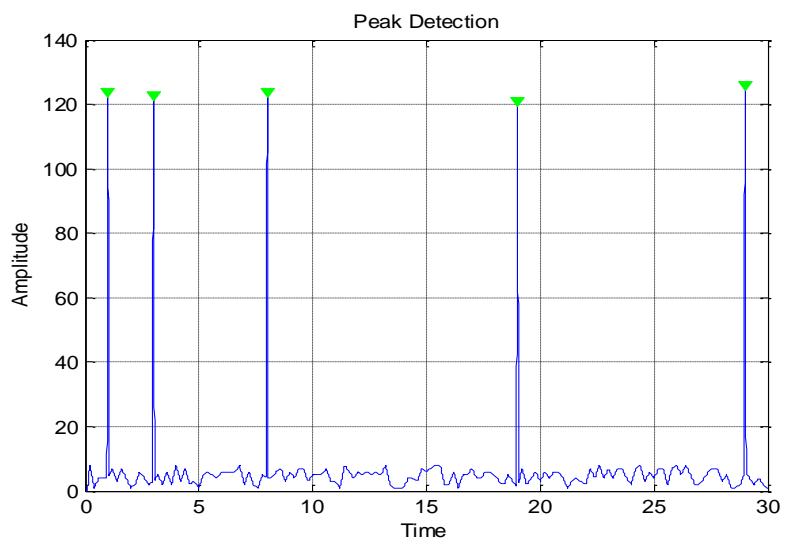

Figure 7. All determined turning point using the standard deviation threshold calculation method

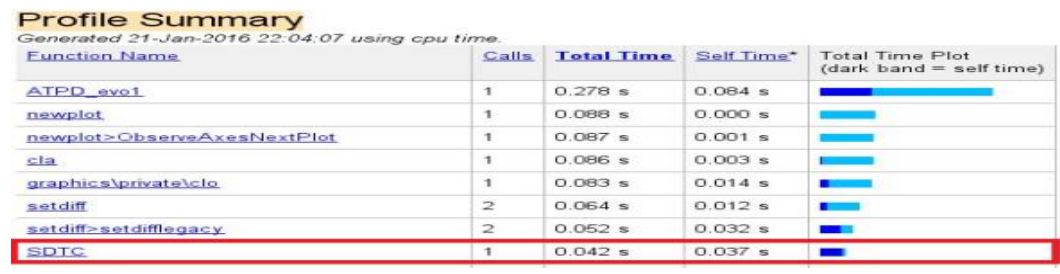

Figure 8 . Profile summary of M-ATPD created by MATLAB simulation 
Table 1. Computational time of M-ATPD compared to other methods

\begin{tabular}{cccc}
\hline & & ATPD $(\mathrm{s})$ & M-ATPD $(\mathrm{s})$ \\
\hline \multirow{2}{*}{ Non-Noise } & Pulse & NA & 0.29 \\
Signal & Sinusoidal & 0.56 & 0.28 \\
& Triangular & 0.58 & 0.29 \\
& Pulse & NA & 0.29 \\
Noise Signal & Sinusoidal & 0.56 & 0.28 \\
& Triangular & 0.56 & 0.28 \\
& Average & 0.57 & 0.29 \\
& Normalize of & 2.0 & 1.00 \\
\hline
\end{tabular}

*NA is refer to non-stop simulation.

Table 2. Detection error of M-ATPD compared to other methods

\begin{tabular}{clcc}
\hline & & ATPD $(\%)$ & M-ATPD $(\%)$ \\
\hline \multirow{2}{*}{ Non-Noise } & Pulse & NA & 33.33 \\
Signal & Sinusoidal & 26.67 & 33.33 \\
& Triangular & 13.33 & 33.33 \\
\multirow{2}{*}{ Noise Signal } & Pulse & NA & 0.00 \\
& Sinusoidal & 26.67 & 13.33 \\
& Triangular & 13.33 & 6.67 \\
\hline
\end{tabular}

*NA is refer to non-stop simulation.

To evaluate the performance of the peak detection algorithm, we use three benchmark parameters, which are positive prediction $(+\mathrm{P})$, sensitivity (SE) and detection error (DER). To calculate $+\mathrm{P}, \mathrm{SE}$ and DER, we use rates such as false negative (FN), which represents the failure to detect a true peak (a peak that is not detected as a peak), and false positive (FP), which refers to false peak detection (a non-peak detected as a peak). By using FN and FP, $+\mathrm{P}, \mathrm{SE}$ and DER can be calculated as shown in (1), (2) and (3), respectively, as suggested by [12]-[15]. TP represents the number of true positive detections (peaks detected as peaks), while TPN represents the total number of peaks in a signal. + P reports the percentage of peak detections that can be regarded as true peaks. SE reports the percentage of true peaks that were correctly detected by the algorithm. DER reports the percentage of peak detection errors by the algorithm.

$$
\begin{aligned}
& +P=\frac{T P}{T P+F P} \\
& S E=\frac{T P}{T P+F N} \\
& D E R=\frac{F P+F N}{T P N}
\end{aligned}
$$

From the results, M-ATPD fixed the error in the non-stop simulation of ATPD for detecting pulse signals. The peak detection error of non-noise sinusoidal signals increases from $26.67 \%$ to $33.33 \%$. The peak detection error of non-noise triangular signals increases from $13.33 \%$ to $33.33 \%$. This shows a slight degradation in the accuracy of peak detection of non-noise signals when comparing M-ATPD to ATPD. The peak detection error of noise sinusoidal signals decreases from $26.67 \%$ to $13.33 \%$. The peak detection error on noise triangular signals increases from $13.33 \%$ to $6.67 \%$. This shows a slight improvement in the accuracy of peak detection of noise signals when comparing M-ATPD to ATPD. Although M-ATPD shows as degradation in the accuracy of detecting non-noise signals, there is improvement in relation to noise signals. This is due to the standard deviation threshold calculation method, which is purposely proposed for noise signals, because standard deviation is used to estimate the noise level. Hence, the standard deviation threshold is used to eliminate the noise peak. When the method is applied to non-noise signals, the accuracy will be lower. However, noise will always exist in real signals. Hence, this method can be applied in real applications. Next, the experiment results of M-ATPD to biosensor signal will be discuss.

Table 3 shows the performance results of M-ATPD using simulation in MATLAB. M-ATPD obtained $100 \%$ for SE and $+\mathrm{P}$ when applied to the ECG, BP, and EEG signals. It therefore achieved a zero percentage DER for these signals. For the Resp(sum) signal, M-ATPD reached 99.13\% SE and 99.27\% +P. It therefore achieved $1.59 \%$ DER for the Resp(sum) signal. The results for ATPD, on the other hand, show better performance for BP, followed by ECG, EEG and Resp(sum). This is because M-ATPD can perform better peak detection on the signal, giving a higher contrast between noise level and peak level. On average, M-ATPD reached $99.95 \% \mathrm{SE}$ and $99.96 \%+\mathrm{P}$, and therefore achieved $0.10 \%$ DER. 
Table 3. Results of M-ATPD

\begin{tabular}{cccccccc}
\hline Signal & TPN & TP & FP & FN & Se\% & + P\% & DER\% \\
\hline ECG & 3846 & 3846 & 0 & 0 & $100.00 \%$ & $100.00 \%$ & $0.00 \%$ \\
BP & 3846 & 3846 & 0 & 0 & $100.00 \%$ & $100.00 \%$ & $0.00 \%$ \\
EEG & 2743 & 2743 & 0 & 0 & $100.00 \%$ & $100.00 \%$ & $0.00 \%$ \\
Resp(sum) & 690 & 684 & 5 & 6 & $99.13 \%$ & $99.27 \%$ & $1.59 \%$ \\
Total & 11125 & 11119 & 5 & 6 & $99.95 \%$ & $99.96 \%$ & $0.10 \%$ \\
\hline
\end{tabular}

Figure 9 shows the results of the experiment for ECG, BP, EEG and Resp(sum) signals respectively. Each figure shows only a 30s signal from the full signal 3600s signal, due to the limited space for

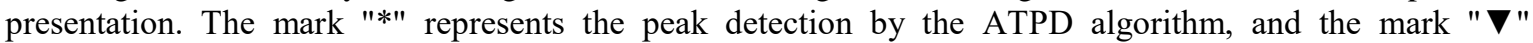
represents the peak detection by M-ATPD. Table 4 presents the computational time results of M-ATPD. These results for computational time are based on a simulation in MATLAB of the computational time of the algorithms. M-ATPD required the shortest time for EMG, followed by BP, ECG and Resp(sum), and these times were $4.80 \mathrm{~s}, 4.96 \mathrm{~s}, 5.32 \mathrm{~s}$ and 5.32s respectively. M-ATPD used an average computational time of 5.10s. The results show that the times required for each signal were approximately equal.
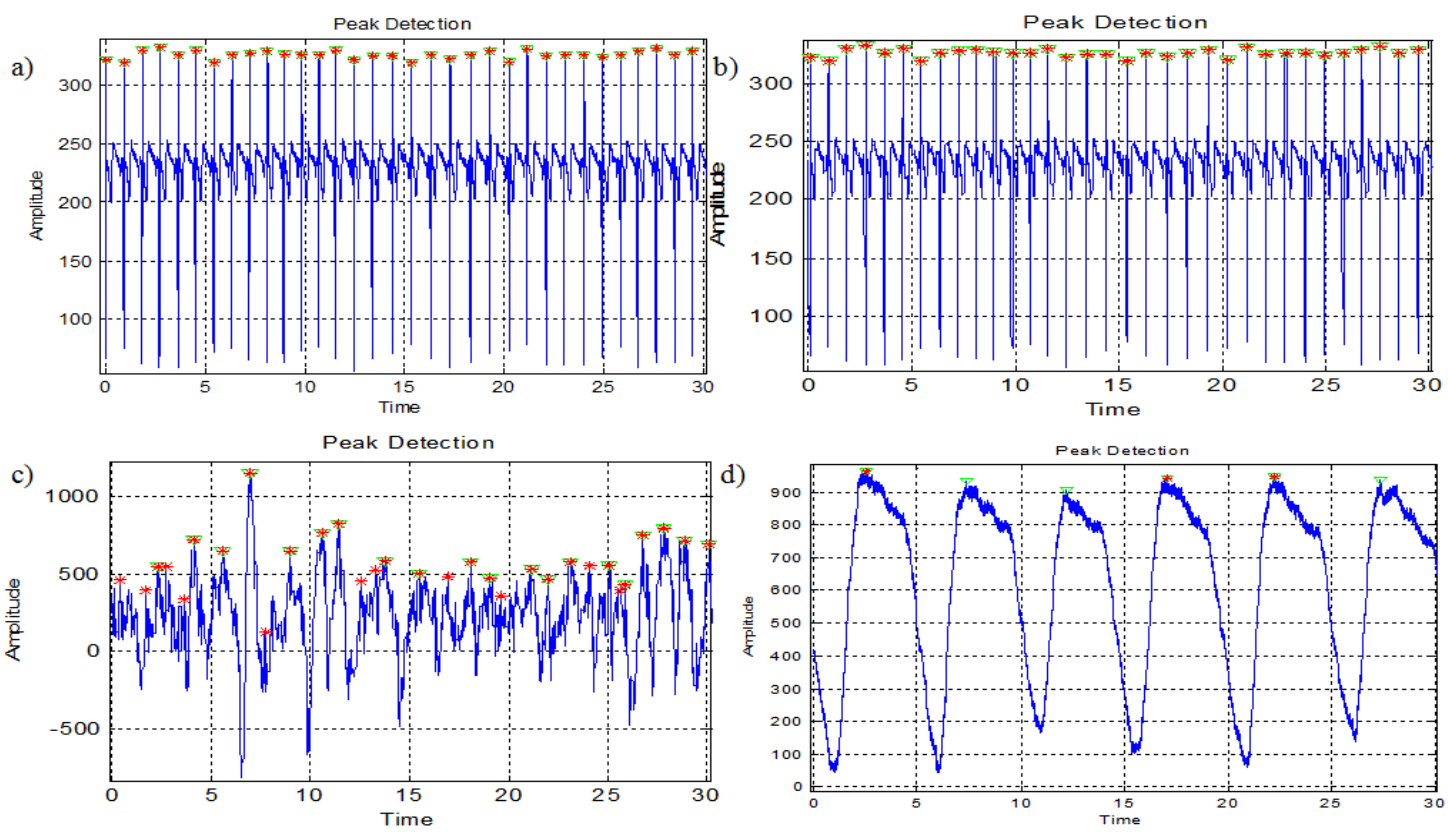

Figure 9. (a) Results of ECG signal (b) Results of BP signal (c) Results of EEG signal (d)Results of Resp(sum) signal

Table 4. Computational time results of M-ATPD

\begin{tabular}{ccccccc}
\hline Signal & 1 & 2 & 3 & 4 & 5 & Average \\
\hline ECG & 5.30 & 5.34 & 5.31 & 5.35 & 5.32 & 5.32 \\
BP & 4.92 & 4.88 & 4.89 & 4.96 & 5.14 & 4.96 \\
EEG & 4.77 & 4.73 & 4.99 & 4.72 & 4.80 & 4.80 \\
Resp(sum) & 5.30 & 5.33 & 5.27 & 5.31 & 5.37 & 5.32 \\
& & Average & & & 5.10 \\
\hline
\end{tabular}

Next, the results of ATPD are presented. Table 5 shows the performance results from ATPD using simulation in MATLAB. ATPD obtained $99.43 \%$ SE and $100 \%+P$ for ECG, hence achieving $0.57 \%$ DER; it reached $99.61 \%$ Se and $100 \%+\mathrm{P}$ for $\mathrm{BP}$, hence achieving $0.39 \% \mathrm{DER}$; it achieved $100 \%$ SE and $57.60 \%+\mathrm{P}$ for EEG, hence achieving $73.61 \%$ DER; and reached $82.73 \%$ SE and $100 \%+\mathrm{P}$ for Resp(sum), hence achieving $16.52 \%$ DER. The results show that ATPD gives best performance for BP, followed by ECG, Resp(sum) and EEG. This is due to the noise level and the signal shape. On average, ATPD achieved 98.64\% SE and $84.44 \%+\mathrm{P}$, thus achieving $19.51 \%$ DER. Table 6 shows the computational time results for ATPD. ATPD required the shortest time for Resp(sum), followed by BP, EMG and ECG, and the times for these 
were $14.75 \mathrm{~s}, 35.87 \mathrm{~s}, 43.73 \mathrm{~s}$ and $82.15 \mathrm{~s}$ respectively, giving an average computational time of $44.13 \mathrm{~s}$. The results show that the time required to process each signal varied widely. This is due to the threshold calculation process requiring a different period of time for each type of signal. Lastly, the results of M-ATPD and ATPD are compared. In terms of DER, M-ATPD has lower DER\% overall, in comparison with ATPD; M-ATPD has only $0.10 \%$ DER, while ATPD has $19.51 \%$ DER. In terms of computational time, M-ATPD required 5.10s on average, while ATPD requires 44.13s. These results show that M-ATPD can perform peak detection on average 8.65times faster than ATPD for the same signal.

Table 5. Results of ATPD

\begin{tabular}{cccccccc}
\hline Signal & TPN & TP & FP & FN & Se\% & + P\% & DER\% \\
\hline ECG & 3846 & 3824 & 0 & 22 & $99.43 \%$ & $100.00 \%$ & $0.57 \%$ \\
BP & 3846 & 3846 & 0 & 15 & $99.61 \%$ & $100.00 \%$ & $0.39 \%$ \\
EEG & 2743 & 2743 & 2019 & 0 & $100.00 \%$ & $57.60 \%$ & $73.61 \%$ \\
Resp(sum) & 690 & 546 & 0 & 114 & $82.73 \%$ & $100.00 \%$ & $16.52 \%$ \\
Total & 11125 & 10959 & 2019 & 151 & $98.64 \%$ & $84.44 \%$ & $19.51 \%$ \\
\hline
\end{tabular}

Table 6. Computational time results of ATPD

\begin{tabular}{ccccccc}
\hline Signal & 1 & 2 & 3 & 4 & 5 & Average \\
\hline ECG & 82.18 & 82.12 & 82.14 & 82.17 & 82.16 & 82.15 \\
BP & 35.90 & 35.84 & 35.84 & 35.87 & 35.88 & 35.87 \\
EEG & 43.72 & 43.74 & 43.73 & 43.75 & 43.72 & 43.73 \\
Resp(sum) & 14.70 & 14.82 & 14.76 & 14.76 & 14.73 & 14.75 \\
& \multicolumn{7}{c}{ Average } & & & 44.13 \\
\hline
\end{tabular}

\section{CONCLUSION}

In this paper, a new algorithm was proposed, which is modification of ATPD. In previous work, ATPD have the better performance among the method mention in Section I. This work continuous to further improve the performance of ATPD by modification. ATPD is modified in order to improve the significant level of time consumption at the threshold calculation stage, while ensuring the accuracy of the algorithm is maintained. To overcome the problem, a new threshold calculation method is proposed, namely, a standard deviation threshold calculation. The results show that the standard deviation threshold calculation reduces time consumption by more than $80 \%$ compared to a cluster threshold calculation. For control signal experiment, when the standard deviation threshold calculation is applied using a peak detection known as MATPD, time consumption is reduced by about 2 times faster compared to ATPD. The detection error of MATPD in relation to noise signals decreases by $6.67 \%$ compared to ATPD. In other words, M-ATPD is a more accurate method for conducting peak detection on noise signals compare to ATPD. For raw signal, four types of biosignal (ECG, BP, EEG and Resp(sum)) were selected in order to investigate the performance of M-ATPD. The results show that the M-ATPD algorithm can achieve $19.41 \%$ lower DER compared with ATPD, and in terms of computational time, M-ATPD can perform 8.65times faster than ATPD. Overall, the results demonstrate that the proposed M-ATPD algorithm gives better performance than ATPD.

\section{ACKNOWLEDGEMENTS}

The authors would like to acknowledge the support from the Fundamental Research Grant Scheme (FRGS) under a grant number of FRGS/1/2014/SG05/UNIMAP/02/3 from the Ministry of Higher Education Malaysia.

\section{REFERENCES}

[1] F. Scholkmann, J. Boss, and M. Wolf, "An Efficient Algorithm for Automatic Peak Detection in Noisy Periodic and Quasi-Periodic Signals,” Algorithms, vol. 5, no. 4, pp. 588-603, Nov. 2012.

[2] Y.-J. Yu, Q.-L. Xia, S. Wang, B. Wang, F.-W. Xie, X.-B. Zhang, Y.-M. Ma, and H.-L. Wu, “Chemometric strategy for automatic chromatographic peak detection and background drift correction in chromatographic data.," $J$. Chromatogr. A, vol. 1359, pp. 262-70, Sep. 2014.

[3] H. S. Shin, C. Lee, and M. Lee, "Adaptive threshold method for the peak detection of photoplethysmographic waveform.," Comput. Biol. Med., vol. 39, no. 12, pp. 1145-52, Dec. 2009.

[4] H. Zhu and J. Dong, "An R-peak detection method based on peaks of Shannon energy envelope," Biomed. Signal Process. Control, vol. 8, no. 5, pp. 466-474, Sep. 2013.

[5] J. Park, J. Song, H. Kim, and D. Ryu, "Peak Detection for Portable Multi-modal Nano-bio Sensor System," Int. J. 
Bio-Science Bio-Technology, vol. 5, no. 3, pp. 135-142, 2013.

[6] A. L. Jacobson, "Auto-threshold peak detection in physiological signals," 2001 Conference Proceedings of the 23rd Annual International Conference of the IEEE Engineering in Medicine and Biology Society, Istanbul, Turkey, 2001, pp. 2194-2195 vol.3.

[7] M. Florian, J. K. Lenstra, and A. H. G. Rinnooy Kan, "Deterministic Production Planning: Algorithms and Complexity Author ( s ): M. Florian, J . K . Lenstra and A . H . G. Rinnooy Kan Published by: INFORMS Stable URL: http://www.jstor.org/stable/2630701 REFERENCES Linked references are available on JST," Manage. Sci., vol. 26, no. 7, pp. 669-679, 1980.

[8] A. E. F. Clementi, P. Crescenzi, P. Penna, G. Rossi, and P. Vocca, "On the Complexity of Computing Minimum Energy Consumption Broadcast Subgraphs," in STACS 2001, Springer Berlin Heidelberg, 2001, pp. 121-131.

[9] A. P. Chandrakasan and R. W. Brodersen, "Minimizing Power Consumption in Digital CMOS Circuits," Proc. IEEE, vol. 83, no. 4, pp. 498 - 523, 1995.

[10] Z. Zhao, J. Yuan, Q. Gao, and Y. Kong, "WAVELET IMAGE DE-NOISING METHOD BASED ON NOISE STANDARD," in Proceedings of the 2007 International Conference on Wavelet Analysis and Pattern Recognition, 2007, pp. 1910-1914.

[11] D. Fang, "Application of Daubechies Wavelet Transform in the Estimation of Standard Deviation of White Noise," in 2011 Second International Conference on Digital Manufacturing and Automation (ICDMA), 2011, vol. 0, pp. $212-215$.

[12] M. Merah, T. A. Abdelmalik, B. H. Larbi, E. M. Naouar, and B. D.- Oran, "R-peaks detection based on stationary wavelet," Comput. Methods Programs Biomed., vol. 121, no. 3, pp. 149-160, 2015.

[13] J. P. Martínez, R. Almeida, S. Olmos, A. P. Rocha, and P. Laguna, "A Wavelet-Based ECG Delineator : Evaluation on Standard Databases," IEEE Trans. Biomed. Eng., vol. 51, no. 4, pp. 570-581, 2004.

[14] V. X. Afonso and W. J. Tompkins, "ECG Beat Detection Using Filter Banks," IEEE Trans. Biomed. Eng., vol. 46, no. 2, pp. 192-202, 1999.

[15] Y. Min, H. Kim, S. Member, Y. Kang, G. Kim, J. Park, and S. Kim, "Design of Wavelet-Based ECG Detector for Implantable Cardiac Pacemakers," IEEE Trans. Biomed. Circuits Syst., vol. 7, no. 4, pp. 426-436, 2013.

\section{BIOGRAPHIES OF AUTHORS}

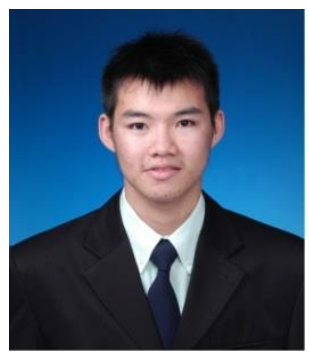

Teh Yi Jun is currently a PhD student in School of Microelectronics Engineering, Universiti Malaysia Perlis. His main research is on nano-bio sensors signal characteristics and its on-chip analysis algorithms for early diseases detection. He has his B. Eng. degree in Microelectronic engineering from Universiti Malaysia Perlis in 2014.

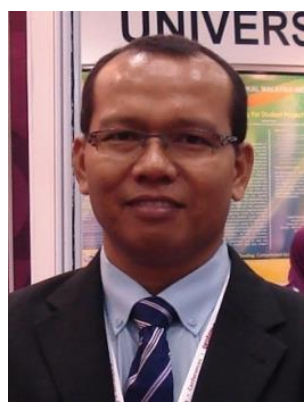

Dr. Asral Bahari is a senior lecturer at the School of Microelectronics Engineering, Universit Malaysia Perlis (UniMAP), and was a Programme Chairperson for the Electronics Engineering Degree Programme, UniMAP, from September 2009 to Mac 2013. He has more than 15 years experience in VLSI design in both the industry and academic sectors, and has been involved at various levels of VLSI design such as transistor modelling, digital circuit design, analogue circuit design, logic synthesis and physical place and route, architecture design and algorithm development.

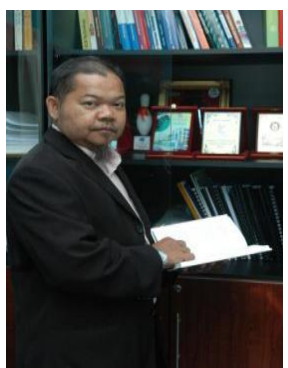

Prof. Dr. Uda Hashim is a Professor at Institute of Nano Electronic Engineering (INEE), Universiti Malaysia Perlis (UniMAP), and was a Director for the INEE, UniMAP since October 2008. He has more than 25 years experience in Semiconductor Devices, CMOS Based Sensor, Nanoelectronic and Nano Biochip in both the industry and academic sectors. 